\title{
A ANÁliSE DOCUMENTAL NA AVALIAÇÃo DE PRÁTICAS EDUCATIVAS EM SAÚDE
}

\author{
Alfredo Almeida PINA-OLIVEIRA ${ }^{1}$ \\ Ana Claudia Camargo Gonçalves GERMANI ${ }^{2}$
}

Anna Maria CHIESA ${ }^{3}$

\begin{abstract}
${ }^{1}$ Enfermeiro. Doutor em Ciências da Saúde. Educador em Saúde Pública do Centro de Promoção da Saúde do Hospital das Clínicas da Faculdade de Medicina da Universidade de São Paulo.

${ }^{2}$ Médica. Doutora em Ciências da Saúde. Professora Doutora do Departamento de Medicina Preventiva da Faculdade de Medicina da Universidade de São Paulo.

${ }^{3}$ Enfermeira. Livre Docente em Enfermagem. Professora Associada da Escola de Enfermagem da Universidade de São Paulo.
\end{abstract}

Endereço para correspondência: Alfredo Almeida Pina-Oliveira. Av. Dr. Enéas de Carvalho Aguiar, 155, $4^{\circ}$ andar, bloco 6, secretaria do Ambulatório Geral e Didático do Hospital das Clínicas da Faculdade de Medicina da Universidade de São Paulo. São Paulo - SP. CEP: 05403-000. E-mail: alfredo.pina@ @h.fm.usp.br.

Fonte de financiamento: não houve.

Recebido em: 01/07/2015 - Aprovado em: 14/01/2016 - Disponibilizado em: 30/07/2016

\section{Resumo}

A documentação contribui para o melhor planejamento, execução e avaliação de processos educativos na área da saúde. Objetivo: discutir os limites e possibilidades da utilização da técnica de análise documental para o registro sistemático das atividades educativas em saúde. Método: ensaio reflexivo sobre o emprego da análise documental na avaliação da capacitação do Projeto 'Nossas Crianças: Janelas de Oportunidades' e com potencial para a sistematização dos registros de processos educativos em saúde em diferentes cenários. Resultados: identificação de recursos, instrumentos e abordagens constitutivos do processo ensino-aprendizagem para a documentação de ações educativas realizadas por profissionais de saúde baseados nos objetivos, conteúdos, recursos físicos, recursos humanos, recursos materiais, estratégias de ensino, periodicidade e carga horária dos encontros, e tipo de avaliação. Conclusões: evidenciou-se a relevância da análise documental na seleção, organização e avaliação dos registros relacionados a práticas educativas que podem contribuir para a qualidade da formação de recursos humanos e da assistência nos serviços de saúde.

Descritores: Pesquisa qualitativa, modelos educacionais, documentação, educação em saúde.

\section{DOCUMENTAL ANALYSIS IN EVALUATIVE HEALTH EDUCATION PRACTICES}

\begin{abstract}
Documentation contributes to plan, develop and evaluate educational processes in healthcare system. Objective: to discuss pros and cons of documental analysis for systematic recording on health education practices. Method: reflexive essay on documentation related to health educators' formation of extension project 'Our Children: Windows of Opportunities' and its potential systematic recording regarding different educational scenarios. Results: identification of protocol, criteria of teaching and learning process and approach for health educators' documentation about health education based on objectives, contents, structure, human resources, instructional materials, teaching strategies, periodicity and class load, and evaluation models. Conclusions: we have evinced an important role of systematic registering focused on selecting, organizing and evaluating educational processes that contribute to human resources formation and quality of assistance in healthcare settings.
\end{abstract}

Keywords: Qualitative research, educational models, documentation, health education. 


\section{Introdução}

O surgimento da escrita trouxe para a humanidade uma segunda possibilidade de transmissão do conhecimento e registro dos fenômenos individuais e sociais, sendo assim um recurso mais estável e perene do que a comunicação oral.

$\mathrm{Na}$ perspectiva de Richardson (1999), documentos são todos os tipos de registros escritos que podem ser usados como fonte de informação sobre $o$ comportamento humano a fim de melhor alcançar a compreensão das relações interpessoais e situações que expressam os momentos de produção (trabalho) e reprodução social (formas de viver).

A análise documental propicia a passagem de um documento primário ou original para um material secundário que é uma representação analítica e ou sintética do primeiro, feita por meio de aproximações que utilizam referenciais teóricos de análise (BARDIN, 2011; BOWLING， 2010; CAREGNATO \& MUTTI, 2006; LIAMPUTTONG \& EZZY, 2005; MINAYO, 2008; POPE \& MAYS, 2005; RICHARDSON, 1999).

Segundo Costa (2011, p. 368), a investigação acerca de processos representa sempre "um conjunto de ações encadeadas em um determinado período histórico e social", necessitando de um determinado levantamento de dados que possibilite a reconstrução dos acontecimentos, das situações e seus antecedentes por meio de documentos originados em políticas de saúde internacionais e nacionais, ações programáticas ou nos próprios processos de trabalho das instituições de saúde, seja no âmbito assistencial (por exemplo, educação em saúde) seja na formação de recursos humanos (por exemplo, programas de educação continuada ou pólos de educação permanente).

Na compreensão de Gil (2008), a pesquisa documental compartilha similaridades com a pesquisa bibliográfica no tocante ao desenvolvimento e tratamento das informações, entretanto dela se diferencia quanto à natureza de determinadas fontes de dados tais como reportagens de jornal, cartas, diários, biografias, elementos iconográficos, contratos, filmes, fotografias, gravações etc.

Nesse sentido, o presente estudo teve como objetivo discutir os limites e possibilidades da utilização da técnica de análise documental para o registro sistemático das atividades educativas em saúde. 


\section{Materiais e método}

A técnica da análise documental consiste em uma série de operações que visam estudar e analisar um ou vários documentos para descobrir as circunstâncias sociais, históricas, culturais e econômicas com as quais o objeto de estudo pode estar relacionado (BARDIN, 2011; BOWLING，2010; CAREGNATO \& MUTTI, 2006; LIAMPUTTONG \& EZZY, 2005; MINAYO, 2008; POPE \& MAYS, 2005).

Tratou-se de um ensaio reflexivo sobre o emprego dessa técnica qualitativa em um processo de formação de multiplicadores do Projeto "Nossas Crianças: Janelas de Oportunidades" (doravante denominado Projeto Janelas) nas equipes da Estratégia Saúde da Família (ESF) no município de São Paulo (CHIESA et al., 2009; OLIVEIRA, 2007).

A etapa de preparação documental, também denominada pré-análise, envolveu uma primeira leitura de diversos documentos coletados que foram primeiramente classificados e indexados (BARDIN, 2011; MINAYO, 2008).

Após serem divididos em documentos principais e complementares, receberam uma determinada codificação. Esses documentos, assim selecionados, classificados, indexados e submetidos a uma primeira leitura, constituíram o corpus do estudo. A análise documental propriamente dita incidiu sobre aqueles documentos classificados como documentos principais, ou seja, o corpus per se (BARDIN, 2011; CAREGNATO \& MUTTI, 2006; MINAYO, 2008; OLIVEIRA, 2007).

Nos documentos principais foram identificadas concepções relacionadas à promoção do desenvolvimento infantil através do fortalecimento dos cuidados familiares apresentadas nos Textos de Apoio (book de alinhamento conceitual disponível em: http://www.ee.usp.br/pesquisa/grupromo/d ocs/projetos/projeto_janelas.pdf) e no Manual "Toda hora é hora de cuidar" (disponível

em: http://www.unicef.org/brazil/pt/manualtod ahora.pdf), documentos esses elaborados pela equipe técnica do Projeto Janelas.

Além disso, os documentos que continham a Programação das Oficinas de Capacitação e a Avaliação dos Participantes permitiram compreender a operacionalização dos conceitos teóricos e metodológicos nas etapas de planejamento, execução e avaliação do processo educativo desses sujeitos (OLIVEIRA, 2007).

Quanto aos documentos complementares, eles foram constituídos por (1) atas de reuniões de 
acompanhamento das ações de implantação do Projeto Janelas, (2) planos de ação elaborados pelos próprios responsáveis pela 'multiplicação' da formação recebida junto às equipes de ESF e (3) relatórios de execução que descrevem a implantação do Projeto Janelas nas regiões norte, sul, leste e centro-oeste da cidade de São Paulo (OLIVEIRA, 2007).

A construção das categorias empíricas com vistas à descoberta de núcleos de sentido com presença ou frequiência significativa no corpus do estudo tem sido amplamente descrita (BARDIN， 2011; MINAYO, 2008; RICHARDSON, 1999).

Nesta etapa, a intenção foi construir um roteiro e encontrar elementos convergentes de diferentes tendências pedagógicas predominantes no ensino brasileiro, a saber: a tradicional, a renovada, a tecnicista e a crítica (OLIVEIRA, 2007) e foram empregados diferentes fundamentações teóricas para a compreensão das interfaces entre saúde e educação (BORDENAVE \& PEREIRA, 2002; FREIRE, 2002; LIBÂNEO, 1994; LUCKESI, 2011; ROMÃO, 2011).

\section{Resultados}

A identificação das relações existentes entre determinados elementos e os temas mais recorrentes nos documentos estudados possibilitou a extração das categorias indicadas e descritas a seguir:

- Objetivos: norteiam as atividades para a obtenção de resultados e oferecem parâmetros para medir, comparar e rever o desempenho obtido. Podem ser gerais ou específicos (BORDENAVE \& PEREIRA, 2002).

Objetivos do Projeto Janelas: (1) Sensibilizar os 'agentes multiplicadores' para a criação de um espaço de capacitação dinâmico, criativo $e$ participativo para o trabalho em equipe; $e$ (2) Multiplicar os conceitos da capacitação no cotidiano dos serviços de saúde a fim de introduzir, de forma competente e motivada, a cartilha da família e a ficha de acompanhamento dos cuidados.

- Conteúdos: segundo Libâneo (1994), conteúdos são conhecimentos, habilidades, valores e atitudes organizados pedagógica e didaticamente com vistas à assimilação ativa pelos alunos em sua prática de vida.

Conteúdos do Projeto Janelas: acolhimento e integração dos participantes; promoção da saúde; importância da primeira infância; proteger e cuidar; desenvolvimento infantil ampliado; família e rede social; função materna e função paterna; papéis 
familiares e a divisão dos cuidados; competências familiares/cuidados essenciais para o desenvolvimento infantil; estimulação e aprendizagem da criança de zero a seis anos; direitos de cidadania e acesso a serviços de saúde, educação, assistência social, sistema de garantia de direitos, entre outros.

- Recursos físicos: referem o tipo de área física onde ocorrem os encontros, sua configuração e arranjos espaciais.

Ambiente físico do Projeto Janelas: uma sala grande com cadeiras dispostas em círculo para realização das plenárias; salas menores para trabalhos em pequenos grupos; ambiente externo facilitador de contato com a natureza.

- Recursos humanos: de acordo com Luckesi (2011), o educador atua como um mediador entre a coletividade da sociedade e a individualidade do educando, dando direção ao ensino e a aprendizagem.

Projeto Janelas: seis facilitadoras, garantindo-se pelo menos uma dupla por encontro (duas enfermeiras, uma psicóloga, duas assistentes sociais e uma administradora); e 130 profissionais de saúde envolvidos com a estratégia Saúde da Família (médicos, enfermeiros, auxiliares de enfermagem, agentes comunitários de saúde, auxiliares administrativos, auxiliares de limpeza, gerentes, supervisores $e$ interlocutores regionais e locais).

- Recursos materiais: são as bases concretas, os insumos que viabilizam a execução das atividades.

Projeto Janelas / material didático: cartilha da família e manual de apoio "Toda hora é hora de cuidar"; ficha de acompanhamento dos cuidados para a promoção da saúde da criança; book de apoio; cópia reprográfica da programação das atividades da oficina de capacitação de 'multiplicadores'; textos avulsos e letras de música.

Recursos audiovisuais: microcomputador ou notebook; datashow; retroprojetor; transparências e canetas apropriadas; flipchart; rádio com toca $C D$ e CDs variados (temáticos e musicais); televisão; vídeo cassete e fitas de vídeo (temáticas e filmes).

\section{- Periodicidade e carga horária dos} encontros: referem-se à organização das atividades no tempo e sua duração. A oficina de capacitação do Projeto Janelas compõe-se de cinco encontros de oito horas cada um, freqüência semanal, totalizando uma carga horária de 40 horas.

- Estratégias de ensino: nesta categoria estão reunidas e descritas as 
estratégias e técnicas empregadas. São bastante diversificadas e devem estar de acordo com os objetivos e os conteúdos do processo educativo, como por exemplo, com o desenvolvimento da capacidade de observação, análise, teorização, síntese e práxis (FREIRE, 2002).

Projeto Janelas: metodologia problematizadora e participativa; técnicas de grupo para acolhimento e integração; apresentação e construção de conteúdos através de exposições dialogadas; leituras compartilhadas e comentadas em pequenos grupos; análises críticas de músicas e filmes; elaboração de sínteses através de cartazes e debates em plenárias.

- Tipo de avaliação: Luckesi (2011) ressalta a importância e a indissociabilidade do planejamento e da avaliação, pois ambos objetivam a obtenção de resultados satisfatórios a partir de meios e recursos propícios ao estabelecimento de situações de aprendizagem adequadas.

Projeto Janelas: utilização de rodas de avaliação oral ao final de cada dia e preenchimento de ficha de avaliação individual ao final da oficina.

\section{Discussão}

A análise documental do processo educativo do Projeto Janelas propiciou a reprodutibilidade dos conteúdos, recursos didáticos e metodologias pelos educadores em seus respectivos locais de atuação (CHIESA et al., 2009; OLIVEIRA, 2007).

A abordagem ou forma de coletar os dados na triangulação pode variar bastante (DRIESSNACK et al., 2007). Esses autores identificam (a) a triangulação de dados (busca a convergência ou corroboração de dados no mesmo fenômeno); (b) a triangulação de investigadores (dois ou mais investigadores colaboram na coleta e análise dos dados); (c) a triangulação teórica (utilização de mais de um arcabouço teórico); e (d) a triangulação metodológica (utilização de métodos mistos ou múltiplos para a coleta e análise de dados).

Em relação à análise documental, Lüdke e André (1986) destacam a riqueza e a estabilidade das informações contidas em documentos, aspecto este que permite analisar repetidas vezes essas "fontes fixas" de dados, assim como sustentar ou ratificar/validar os resultados obtidos em outros estudos.

Importante observar também que a observação documental não tem como objeto os fenômenos sociais, quando e 
como eles ocorrem, mas as manifestações contidas nos registros de tais fenômenos e ideias subjacentes à elaboração dos mesmos, valorizando assim a historicidade presente na elaboração, construção e registro das atividades humanas pertencentes ao devir em sociedade (GIL, 2008).

O custo, em geral bastante baixo, da análise documental configura uma vantagem adicional do método (LUDKE \& ANDRE, 1986; RICHARDSON, 1999). Sua realização depende do tempo e da elucubração atenta do pesquisador na escolha e seleção dos dados mais significativos para a concretização dos seus objetivos de pesquisa.

A análise documental também tem um caráter não-reativo, pois permite a obtenção dos dados quando o acesso ao sujeito é impraticável (pela sua morte, por exemplo) ou quando se faz necessário controlar possíveis alterações nos comportamentos ou visões de mundo dos sujeitos pesquisados (LUDKE \& ANDRE, 1986; RICHARDSON, 1999).

Por se tratar de uma técnica não obstrutiva, isto é, que não altera o ambiente nem tampouco os sujeitos estudados, as "fontes de papel", como Gil (2008) as denomina, muitas vezes são capazes de proporcionar ao pesquisador dados suficientemente ricos, evitando assim a perda de tempo com deslocamentos em pesquisas de campo.

Ao lado dos aspectos positivos até aqui considerados, também é necessário ponderar acerca de seus aspectos limitadores. $\mathrm{O}$ primeiro deles refere-se ao fato de a análise documental se constituir em amostra não representativa dos fenômenos que se pretende estudar. Os registros de atividades, experiências e resultados obtidos nos diferentes espaços de produção social não conseguem contemplar todos os elementos do cotidiano; sendo assim, perpetuam de modo esparso e pouco representativo as situações vividas (LUDKE \& ANDRE, 1986).

No nosso exemplo decorrente do processo de capacitação de multiplicadores do Projeto Janelas, esse viés foi controlado por meio de entrevista com uma informante-chave e uma análise das fichas de avaliação preenchidas pelos participantes dessa formação nos serviços de saúde (OLIVEIRA, 2007).

Um segundo limite freqüentemente apontado por aqueles que são contrários à pesquisa qualitativa, ou por aqueles outros que não vislumbram a complementaridade entre as dimensões quantitativa e qualitativa, refere-se à sua aparente falta de objetividade e validade (CAREGNATO \& 
MUTTI, 2006; DRIESSNACK et al., 2007; DEMO, 2006).

Para contornar esse tipo de limitação, com as categorias empíricas identificadas foi construído um instrumento específico para a coleta dos dados, o que possibilitou complementar a dimensão qualitativa pela quantitativa, aprimorando conseqüentemente a compreensão dos fenômenos estudados. Trata-se de uma técnica relevante para estudos de natureza qualitativa e métodos mistos e potencializa-se por meio da triangulação de métodos (DRIESSNACK et al., 2007).

Por último, tem sido apontado como um limite da análise documental o fato do pesquisador escolher previamente os pontos ou aspectos que ele deseja enfocar nos documentos. Contudo, a questão norteadora e o objeto de investigação estudados em educação devem revelar o posicionamento ético e político dos sujeitos envolvidos na pesquisa (DEMO, 2006).

\section{Considerações finais}

As potencialidades do emprego da análise documental consistem em: facilidade em integrar a análise documental na triangulação de dados e na triangulação de métodos ou técnicas; informações ricas, estáveis e contextualizadas (historicidade dos registros); baixo custo; tempo reduzido para obtenção da informação; caráter não reativo; técnica não obstrutiva; descrição da estrutura, processo e resultados decorrentes das práticas educativas registradas nos serviços de saúde.

As principais limitações da técnica qualitativa correspondem à constituição de amostra não representativa dos fenômenos que se pretende estudar, à falta de objetividade e ao estabelecimento de categorias analíticas previamente escolhidas.

Espera-se que as reflexões contidas neste artigo contribuam para o avanço da sistematização das práticas educativas em saúde de uma maneira geral, e da enfermagem em particular, sensibilizando os profissionais da saúde para a necessidade de se registrar os processos de trabalho, superando assim as formas intuitivas e reprodutivas de modelos educacionais mecanicistas pré-existentes.

Os registros documentais do processo de capacitação do Projeto Janelas indicam que seus participantes incorporaram o papel de 'agentes multiplicadores' das experiências e conhecimentos adquiridos durante a oficina, tendo como foco o aprimoramento de competências e a incorporação da clínica ampliada por meio 
de processos educativos participativos e transformadores.

$$
\text { Conclui-se que os documentos }
$$
produzidos por agentes formadores e gestores de recursos humanos (planos e cronogramas de atividades educativas, manuais técnicos, fichas de avaliação etc.) são valiosos porque (a) favorecem a reprodutibilidade em outros cenários; (b) contribuem para o enriquecimento dos dados de pesquisa e avaliação dos serviços de saúde; (c) compõem material empírico para complementar e validar dados, ampliando assim os limites de outros métodos qualitativos e ou quantitativos de investigação; (d) sistematizam boas práticas proporcionadas pelos processos educativos em saúde nos diferentes níveis de atenção.

\section{Conflitos de interesse}

Os autores declaram não haver conflitos de interesse.

\section{Contribuição dos autores}

AAPO participou da concepção, delineamento e interpretação dos dados da análise documental e redação do artigo. ACCGG e AMC contribuíram com a revisão crítica do artigo e a aprovação da versão a ser publicada.

\section{Referências}

\section{BARDIN, L. Análise de conteúdo.}

Lisboa: Edições 70, 2011.

BORDENAVE, J. E. D.; PEREIRA, A. $M$. Estratégias de ensinoaprendizagem. 24. ed. Petrópolis: Vozes, 2002.

\section{BOWLING, A. Research methods in}

health: investigating health and health services. 3. ed. New York: Mc Graw Hill, 2010 .

CAREGNATO, R. C. A.; MUTTI, R. Pesquisa qualitativa: análise de discurso versus análise de conteúdo. Texto $\&$ Contexto Enfermagem, v. 15, n. 4, p. 679-684, 2006. Disponível em: http://www.scielo.br/pdf/tce/v15n4/v15n 4a17. Acesso em: 01 jul. 2015.

CHIESA, A. M., et al. A construção de tecnologias de atenção em saúde com base na promoção da saúde. Rev. Esc. Enferm. USP, v. 43, n. 2, p. 1352-1357, 2009. Disponível em: http://www.scielo.br/pdf/reeusp/v43nspe 2/en_a36v43s2.pdf. Acesso em: 01 jul. 2015 .

COSTA, M. C. C. Sociologia: introdução à ciência da sociedade. 4. ed. São Paulo: Moderna, 2011.

FREIRE, P. Pedagogia do oprimido: saberes necessários à prática educativa. $33^{\mathrm{a}}$ ed. Rio de Janeiro: Paz e Terra; 2002.

GIL, A. C. Métodos e técnicas de pesquisa social. 6. ed. São Paulo: Atlas, 2008.

LIAMPUTTONG, P.; EZZY, D. Qualitative Research Methods. 2. ed. Victoria: Oxford University Press, 2005.

LIBÂNEO, J. C. Didática. São Paulo: Cortez, 1994. 
LUCKESI, C. C. Avaliação da aprendizagem escolar: estudos e proposições. 22. ed. São Paulo: Cortez Editora, 2011.

LÜDKE, M.; ANDRÉ, M. E. D. A. Pesquisa em educação: abordagens qualitativas. São Paulo: EPU, 1986.

MINAYO, M. C. S. O desafio do conhecimento: pesquisa qualitativa em saúde. 11. ed. São Paulo: Hucitec, 2008.

OLIVEIRA, A. A. P. Análise

documental do processo de capacitação dos multiplicadores do projeto "Nossas Crianças: Janelas de Oportunidades" no município de São Paulo à luz da promoção da saúde. 2007. Dissertação (Mestrado em Enfermagem) - Escola de Enfermagem, Universidade de São Paulo, São Paulo. Disponível em:

http://www.teses.usp.br/teses/disponiveis /7/7137/tde-17052007-101339/pt-br.php. Acesso em: 01 jul. 2015.

POPE, C.; MAYS, N. Pesquisa

qualitativa na atenção à saúde. 2 . ed. Porto Alegre: Artmed, 2005.

RICHARDSON, R. J. Pesquisa social: métodos e técnicas. 3. ed. São Paulo: Atlas, 1999.

ROMÃO, J. E. Avaliação dialógica:

desafios e perspectivas. 9. ed. São Paulo:

Cortez Editora, 2011.

DRIESSNACK, M.; SOUSA, V. D.;

MENDES, I. A. C. Revisão dos desenhos de pesquisa relevantes

para enfermagem: parte 3: métodos mistos e múltiplos. Rev. Latino-Am.

Enfermagem, v. 15, n. 5, 2007.

Disponível

em: http://www.scielo.br/pdf/rlae/v15n5/

pt_v15n5a24.pdf. Acesso em: 01 jul.

2015.
DEMO, P. Pesquisa: princípio científico e educativo. 12. ed. São Paulo: Cortez Editora, 2006. 